\title{
Gout disease-specific quality of life and the association with gout characteristics
}

This article was published in the following Dove Press journal:

Patient Related Outcome Measures

25 March 2010

Number of times this article has been viewed

\author{
Jan D Hirsch ${ }^{1,4}$ \\ Robert Terkeltaub ${ }^{4}$ \\ Dinesh Khanna ${ }^{5}$ \\ Jasvinder Singh ${ }^{6}$ \\ Andrew Sarkin ${ }^{2}$ \\ Micki Shieh² \\ Arthur Kavanaugh ${ }^{3}$ \\ Susan J Lee Le $^{3,4}$ \\ 'Skaggs School of Pharmacy \\ and Pharmaceutical Sciences, \\ ${ }^{2}$ Health Services Research, \\ ${ }^{3}$ Center for Innovative Therapy, \\ Division of Rheumatology, Allergy, \\ and Immunology, University of \\ California San Diego, La Jolla, \\ CA, USA; ${ }^{4}$ Veterans Administration \\ San Diego Healthcare System, La \\ Jolla, CA, USA; ${ }^{5}$ Department of \\ Medicine/Rheumatology, David Geffen \\ School of Medicine, University of \\ California Los Angeles, Los Angeles, \\ CA, USA; ${ }^{6}$ Department of Medicine/ \\ Rheumatology, Minneapolis VA \\ Healthcare System, Minneapolis \\ MN, USA
}

Correspondence: Jan D Hirsch

University of California, San Diego, Skaggs

School of Pharmacy and Pharmaceutical

Sciences: PSB Room 3256, 9500 Gilman

Drive, Mail code 07 I4, La Jolla,

CA 92093-07। 4, USA

$\mathrm{Tel}+\mathrm{I} 8588225562$

Fax + I 8588226857

Email janhirsch@ucsd.edu
Purpose: Assess the association of gout characteristics with health-related quality of life (HRQoL) using a new gout-specific HRQoL instrument, the Gout Impact Scale (GIS).

Patients and methods: Gout patients completed the GIS (five scales [0-100 score each] representing impact of gout overall [three scales] and during an attack [two scales]) and other questions describing recent gout attacks, treatment, gout history, comorbidities, and demographics. Physicians confirmed gout diagnosis, presence of tophi, and most recent serum uric acid (sUA) level. Relationships between gout characteristics and GIS scores were examined using analysis of variance and correlation analyses.

Results: The majority of patients were male $(90.2 \%)$ with a mean age of $62.2( \pm 11.8)$ years. Approximately one-half (49.7\%) reported $\geq 3$ gout attacks in the past year and the majority $(57.9 \%)$ reported experiencing gout-related pain between attacks. Patients had appreciable concern about their gout ("gout concern overall" scale, 63.1 \pm 28.0 ) but believed their treatment was adequate ("unmet gout treatment need" scale (38.2 \pm 21.4$)$ below scale mid-point). Significantly worse GIS scores were associated with increasing attack frequency and greater amount of time with pain between attacks (most scales, $P<0.001$ ). Common objective measures such as sUA level, presence of tophi and the number of joints involved in a typical attack did not appear to be good indicators of the impact of gout on the patients' HRQoL.

Conclusion: Attack frequency and gout pain between attacks were important correlates of patients' ratings of gout impact on their HRQoL. Further studies are needed to determine the minimal important difference for each GIS scale and interpret our results relative to other patient populations with gout.

Keywords: Gout impact scale, GIS, patient-reported outcomes

\section{Introduction}

Gout is a debilitating disease whose prevalence appears to be increasing worldwide due to many factors (eg, aging populations, changes in diet and lifestyle). ${ }^{1}$ In the United States, approximately $2 \%$ of people aged 45 to 65 years and $3 \%$ of people aged over 65 years report having gout. ${ }^{2}$ Although the physical impact of an acute attack may be intuitive, other disease characteristics may also contribute to the disease burden from the patient's perspective. In a recent clinical trial, approximately half of patients reported, at baseline, that during an attack gout interfered with their movement, work, recreational activities, and enjoyment of life. ${ }^{3}$ Almost half (43\%) of patients reporting interference during an attack also indicated they experienced gout-related pain between acute attacks. The presence of tophi has been associated with greater physical functioning disability in gout patients. ${ }^{4-6}$ In a sample of chronic stable gout

submit your manuscript $\mid$ www. dovepress.con 
patients, those who rated gout as their main health concern assigned greater disutility to gout and were willing to pay more money each month for a cure than patients for whom gout was not their main health concern. ${ }^{7,8}$ However, the relationship among serum urate acid (sUA) level, frequency of gout attacks, and health-related quality of life (HRQoL) was viewed as uncertain during a recent regulatory agency review of a new drug application for a gout prophylactic medication. ${ }^{9}$

The importance of assessing patient-reported outcomes (PROs) within gout clinical trials has been recognized and HRQoL has been included in the list of important domains rheumatologists should address in gout-related studies. ${ }^{10,11}$ However, only a few published studies have measured the association of gout characteristics with patients' HRQoL. Compared to the general population, patients with severe gout have been shown to have reduced HRQoL using the Short Form-36 (SF-36) instrument. ${ }^{12,13}$ In addition, patients with chronic stable gout have also reported lower HRQoL (SF-36) compared to the US norm. ${ }^{14}$ Gout patients have also reported lower SF-36 scores, particularly in physical function, compared to nongout patients who had one or more self-reported musculoskeletal conditions. ${ }^{15}$ Similarly, gout patients reported lower overall HRQoL, as measured by World Health Organization's WHOQOL-BREF instrument, than matched control patients with other chronic conditions in a case - control study in the United Kingdom. ${ }^{16}$ In this study the association between gout and overall HRQoL was weaker after controlling for comorbidities, however the association between gout and reduced HRQoL in the physical domain remained significant. Finally, a study of US veterans in the upper Midwest region found the physical domain of HRQoL (SF-36) was lower for patients with gout compared to patients without gout, though this was largely related to comorbidities and sociodemographic characteristics. ${ }^{17}$

Only two studies have investigated the effect of specific gout characteristics on HRQoL. Sundy and colleagues reported significantly lower physical component summary scores (PCS of the SF-36) for patients with tophi, history of gout attacks and high sUA levels ( $>9 \mathrm{mg} / \mathrm{dL}$ ) HRQoL. ${ }^{12}$ However, no adjustment was made for comorbidities or other factors which could contribute to HRQoL. Lee and colleagues reported after adjusting for age, gender, and comorbidities, the number of joints involved during an attack and the frequency of attacks had a significant association with patient's physical and mental component summary scores (PCS and MCS) of the SF-36. ${ }^{14}$
Available studies have used general or generic HRQoL instruments, not gout-specific HRQoL instruments. Diseasespecific HRQoL instruments provide more information than general instruments about the impact of a disease and its treatment from the patient's perspective..$^{18}$ A gout-specific HRQoL instrument, the Gout Impact Scale (GIS) of the Gout Assessment Questionnaire 2.0 $\left(\mathrm{GAQ}_{2.0}\right)$, has recently been developed and validated in a community setting. ${ }^{19}$ As a disease-specific instrument, the GIS may be more sensitive to the differential impact of frequency of gout attacks and other gout specific characteristics on HRQoL than general HRQoL instruments such as the SF36.

There is growing evidence that gout patients have a reduced HRQoL but little is known about the association of specific gout characteristics with HRQoL. Knowing which gout characteristics are associated with patients' HRQoL could help guide patient care decisions as well as new drug evaluations. The objectives of this study were to assess the association of gout-specific characteristics with HRQoL using a new gout-specific HRQoL instrument (GIS).

\section{Methods}

The study was conducted in three US cities: San Diego, Cincinnati, and Minneapolis. The sample was recruited from gout patients attending a variety of clinics (eg, family practice, internal medicine, rheumatology), using physician in-office recruitment, patient response to clinic posters, and local newspaper advertisements. Patients across a broad range of gout severity were recruited. Inclusion criteria were: age between 18 and 85 years, history or presence of gout as determined by a physician, ability to read and/or understand patient-informed consent and independently complete questionnaires in English, and provision of contact information for physician currently treating gout. Physicians of participating patients were contacted via fax to confirm gout diagnosis (date and method), presence or absence of tophi, and most recent serum uric acid level.

All consenting patients completed the $\mathrm{GAQ}_{2.0}$ which contains the GIS. ${ }^{19}$ The GIS allows patients to describe the impact of gout on their HRQoL, while the remaining $\mathrm{GAQ}_{2.0}$ modules allow patients to describe their gout overall (eg, recent gout attacks, treatment, gout history, demographics). The 24-item GIS is comprised of five scales representing the impact of gout overall ("gout concern overall", "gout medication side effects", "unmet gout treatment need") and during an attack ("well-being during attack" and "gout concern during attack"). Example items for each scale are presented in Table 1. Response options for the 
Table I Example Gout Impact Scale (GIS) items

\begin{tabular}{|c|c|}
\hline GIS items & Example items \\
\hline Gout concern overall & $\begin{array}{l}\text { - I am worried that I will have } \\
\text { a gout attack within the next year } \\
\text { - I feel anxious that my gout will } \\
\text { interfere with my future activities }\end{array}$ \\
\hline Gout medication side effects & $\begin{array}{l}\text { - I am bothered by side effects } \\
\text { from my gout medications } \\
\text { - I worry about the long-term } \\
\text { effects of my gout medications }\end{array}$ \\
\hline Unmet gout treatment need & $\begin{array}{l}\text { - I have control over my gout } \\
\text { - My current medications do not } \\
\text { work well to prevent gout attacks } \\
\text { from happening }\end{array}$ \\
\hline Well being during attack & $\begin{array}{l}\text { - How much of the time did you } \\
\text { experience difficulty working } \\
\text { because of your gout symptoms? } \\
\text { - How much did your symptoms } \\
\text { interfere with your enjoyment } \\
\text { of life? }\end{array}$ \\
\hline Gout concern during attack & $\begin{array}{l}\text { - I am mad or angry when I } \\
\text { experience a gout attack } \\
\text { - I miss planned or important } \\
\text { activities when I have a gout attack }\end{array}$ \\
\hline
\end{tabular}

Notes: All items, except those in the "Well being during attack" scale, preceded with "Please indicate how much you agree or disagree with the following statement". "Well being during attack" items were preceded by "During your last attack."

GIS were five-point Likert-type scales (eg, strongly agree to strongly disagree; all of the time to none of the time). GIS Scales were scored from 0 to 100 , with higher scores on each scale indicating "worse condition" or "greater gout impact." Scales were scored if responses were available for at least half of the scale items. Gout characteristics studied included three attack-related (attack frequency, attack pain and number of involved joints in attack) and three regarding gout overall (time with gout pain between attacks, presence of tophi and sUA). Gout characteristics (all except presence of tophi and sUA) were self-reported using questions developed for this study but which were based on questions that had been used in gout clinical trials (eg, the pain questions were answered using a 10 point Visual Analogue Scale [VAS] as is common in rheumatology practice). The characteristics included were selected based on results of previous published studies and clinical judgment supporting these as important correlates to HRQoL levels in gout patients. Response options for non-GIS questions were multiple choice, VAS and Likerttype scales. Questionnaires were completed either during a regularly scheduled clinic visit or at the patient's home and returned via mail. All study procedures were approved by the University of California San Diego, San Diego Veterans Administration Medical Center (VAMC), Cincinnati VAMC,
University of Cincinnati, and Minneapolis VAMC Human Research Protection Programs.

\section{Data analysis}

For descriptive statistics, frequencies were computed for all variables. Means and standard deviations were further determined for continuous variables and scales. Pearson correlation analyses were conducted to examine the relationships between continuous gout characteristic variables and GIS scores. Analyses of variance (ANOVAs) were used to investigate differences in mean GIS scores among groups of patients defined by categorical gout characteristic variables. Partial correlation coefficients were used to examine the association between gout characteristics and GIS scores, controlling for age, comorbidities, and gender. Statistical significance was set at $P<0.05$ and correlations less than 0.29 were considered to be small, between 0.30 and 0.49 were moderate, and greater than 0.5 were large. ${ }^{20}$ Analyses were performed using SPSS software (version 15.0; SAS Institute, Cary, NC, USA).

\section{Results}

A total of 371 patients were enrolled in the study. Sixty-three patients were lost to follow-up and/or did not complete the study leaving 308 patients for analysis. The majority of patients were male $(90.2 \%)$ and Caucasian $(75.9 \%)$ with a mean age of $62.2( \pm 11.75)$ years (Table 2$)$. The most frequently reported comorbidities were hypertension $(74.0 \%)$, hyperlipidemia (58.9\%), kidney problems (35.6\%), and diabetes $(32.4 \%)$. Approximately $60 \%$ of patients were being treated by a rheumatologist for their gout while $38.1 \%$ received their gout care from a primary care provider. Virtually all (92.6\%) patients had prescribed medication for gout attack treatment and/or prevention.

Patient attack and overall gout characteristics varied widely (Table 3 ). Approximately one-half (49.7\%) of patients had experienced three or more gout attacks in the past year. A typical gout attack involved $5.2 \pm 6.9$ joints with a mean pain rating of $6.7 \pm 2.6$ (VAS 0 "no pain" - 10 "pain as bad as can imagine"). The majority (57.9\%) of patients reported experiencing gout-related pain from "a little" to "all of the time" between attacks. Tophi were present in $26 \%$ of patients and the mean sUA was $7.1 \pm 1.9 \mathrm{mg} / \mathrm{dL}$. Patients rated their overall gout severity as a mean of $5.4 \pm 3.2$ (VAS 0 "not severe at all" - 10 "gout as severe as can imagine").

GIS scale scores revealed patients had appreciable concern about their gout overall ("gout concern overall", 63.1 \pm 28.0 ) but believed their treatment was adequate as evidenced by the 
Table 2 Patient characteristics

\begin{tabular}{|c|c|c|}
\hline Study patients $(n=308)$ & $\mathbf{n}$ & $\begin{array}{l}\text { Number of } \\
\text { respondents (\%) }\end{array}$ \\
\hline Gender [n (\%)] & 297 & \\
\hline Male & & $268(90.2 \%)$ \\
\hline Race [n (\%)] & 290 & \\
\hline White & & $220(75.9 \%)$ \\
\hline Black or African American & & $37(12.8 \%)$ \\
\hline Asian & & $16(5.5 \%)$ \\
\hline Other & & $17(5.8 \%)$ \\
\hline Age (years) & 295 & \\
\hline Mean $( \pm S D)$ & & $62.2(11.7)$ \\
\hline \multicolumn{3}{|l|}{ Comorbidities [n (\%) Yes] } \\
\hline Hypertension & 292 & $216(74.0 \%)$ \\
\hline Hyperlipidemia & 292 & $172(58.9 \%)$ \\
\hline Kidney problems & 278 & $99(35.6 \%)$ \\
\hline Diabetes & 287 & $93(32.4 \%)$ \\
\hline Heart attack or heart failure & 286 & 74 (25.9\%) \\
\hline Kidney stones & 282 & $62(22.0 \%)$ \\
\hline Kidney transplant & 285 & $8(2.8 \%)$ \\
\hline Provider specialty [n (\%)] & 210 & \\
\hline Gout treated by rheumatologist & & 124 (59.1\%) \\
\hline $\begin{array}{l}\text { Gout treated by primary care } \\
\text { providers }\end{array}$ & & $80(38.1 \%)$ \\
\hline Other & & $6(2.9 \%)$ \\
\hline $\begin{array}{l}\text { Type of medication } \\
\text { prescribed [n (\%)] }\end{array}$ & 283 & \\
\hline $\begin{array}{l}\text { Medication prescribed } \\
\text { (for attacks and/or prevention) }\end{array}$ & & $262(92.6 \%)$ \\
\hline None prescribed now & & 21 (7.4\%) \\
\hline
\end{tabular}

Note: Differences in number of patients are due to missing responses.

"unmet gout treatment need" score $(38.2 \pm 21.4)$ below the scale mid-point (50) (Table 4). Although the remaining three GIS scales ("gout medication side effects", "gout concern during attack" and "well being during attack") had mean scores near the scale midpoint $(48.3 \pm 25.7,50.2 \pm 24.1$ and $55.8 \pm 26.1)$ the standard deviations for each were approximately one-half of the mean for each indicating patients in this sample had a wide range of scores on each scale.

All GIS scale scores were moderately and positively correlated with patients' rating of their overall gout severity ( $r=0.309$ to $r=0.454)$. The highest correlations were observed for the "gout concern overall" and "gout concern during attack" scales ( $r=0.454$ and 0.445 , respectively).

Of the three attack-related characteristics (attack frequency, attack pain, and number of involved joints in attack), frequency (number of attacks in past year) showed the most consistent relationship with GIS scores (Table 5). Significant differences in GIS scores $(P<0.001$ for all scales
Table 3 Patient gout characteristics

\begin{tabular}{|c|c|c|}
\hline Study patients $(n=308)$ & $\mathbf{n}$ & $\begin{array}{l}\text { Number of } \\
\text { respondents (\%) }\end{array}$ \\
\hline \multicolumn{3}{|l|}{ Attack characteristics } \\
\hline $\begin{array}{l}\text { Number of attacks in the } \\
\text { past year }[\mathrm{n}(\%)]\end{array}$ & 296 & \\
\hline Zero & & $58(19.6 \%)$ \\
\hline $1-2$ & & $91(30.7 \%)$ \\
\hline $3-5$ & & $86(29.1 \%)$ \\
\hline $6-10$ & & $25(8.4 \%)$ \\
\hline$>10$ & & $36(12.2 \%)$ \\
\hline $\begin{array}{l}\text { Gout pain typical attack } \\
(\text { VAS } 0-10) \\
\text { Mean }( \pm S D)\end{array}$ & 161 & $6.7(2.6)$ \\
\hline $\begin{array}{l}\text { Number of joints involved } \\
\text { in Typical attack } \\
\text { Mean }( \pm S D)\end{array}$ & 169 & $5.2(6.9)$ \\
\hline \multicolumn{3}{|l|}{ Overall gout characteristics } \\
\hline $\begin{array}{l}\text { Time with gout pain } \\
\text { between attacks [n (\%)] }\end{array}$ & 285 & \\
\hline All of the time & & $20(7.0 \%)$ \\
\hline Most of the time & & $28(9.8 \%)$ \\
\hline Some of the time & & $58(20.4 \%)$ \\
\hline A little of the time & & $59(20.7 \%)$ \\
\hline None of the time & & $120(42.1 \%)$ \\
\hline Presence of tophi [n (\%Yes)] & 169 & $44(26.0 \%)$ \\
\hline $\begin{array}{l}\text { Latest serum uric acid } \\
\text { (mean } \pm S D)\end{array}$ & 168 & $7.1 \mathrm{mg} / \mathrm{dL} \pm 1.9$ \\
\hline $\begin{array}{l}\text { Overall gout severity } \\
\text { (self-reported VAS 0-10) }\end{array}$ & 260 & \\
\hline Mean $( \pm S D)$ & & $5.4(3.2)$ \\
\hline Range & & $0.10-10.0$ \\
\hline
\end{tabular}

Notes: Differences in number of patients due to missing responses (number of attacks in the past year, time with pain between attacks, overall gout severity), lack of attack occurrences in the past three months (gout pain, number of involved joints, only in patients with an attack in the past three months) and lower number of patients with physician-supplied data (presence of tophi and sUA level).

Abbreviations: SD, standard deviation; sUA, serum uric acid; VAS, Visual Analogue Scale.

except "well-being during attack") were detected among groups of patients categorized by attack frequency, with a significant linear trend for GIS scores to increase with greater attack frequency $(P \leq 0.001)$. Gout pain during a typical attack was moderately correlated with "gout concern overall" $(r=0.301)$ and the two during attack GIS scales ("well being during attack" and "gout concern during attack", $r=0.494$ and $r=0.308$, respectively). Only minimal to small correlations were observed for the number of joints involved in a typical attack and GIS scales $(r=0.184-0.280)$.

Of the three overall gout characteristics studied, time with gout-related pain between attacks showed the most 
Table 4 Gout impact scale scores

\begin{tabular}{lll}
\hline & Mean & SD \\
\hline Overall & & \\
Gout concern overall $(\mathrm{n}=294)$ & 63.1 & 28.0 \\
Gout medication side effects $(\mathrm{n}=294)$ & 48.3 & 25.7 \\
Unmet gout treatment need $(\mathrm{n}=290)$ & 38.2 & 21.4 \\
During attack & & \\
Well being during attack $(\mathrm{n}=290)$ & 55.8 & 26.1 \\
Gout concern during attack $(\mathrm{n}=294)$ & 50.2 & 24.1 \\
\hline
\end{tabular}

Notes: Scale: 0 to 100; higher scores indicate greater gout impact or worse condition. Abbreviation: SD, standard deviation.

consistent relationship with GIS scores (Table 5). Significant differences in GIS scores (all scales, $P<0.001$ ) were detected among groups of patients categorized by amount of time experiencing gout-related pain between attacks in the expected direction (ie, greater time with gout-related pain between attacks was associated with higher [greater gout impact] GIS scores, $P<0.001$ for linear trend). A significant difference between patients with and without tophi was only observed for one GIS scale, "unmet gout treatment need" $(P=0.029)$, with patients with tophi reporting greater (worse) GIS scores. Correlations between sUA level and GIS scales were minimal $(r<0.29)$.

After controlling for age, gender and comorbidities the number of attacks in past year and time with gout pain between attacks remained moderately correlated with the "gout concern overall" ( $r=0.483$ and $r=0.467$, respectively) and unmet treatment need ( $r=0.411$ and $r=0.346$, respectively) (Table 6). Gout pain during a typical attack remained moderately correlated with "gout concern overall" $(r=0.318)$ and gout concern during an attack $(r=0.308)$ while a large

Table 5 Relationships between gout impact scales and gout characteristics

\begin{tabular}{|c|c|c|c|c|c|}
\hline $\begin{array}{l}\text { Study patients }(n=308) \\
\text { Mean }(\mathrm{SD}) P \text { value or } r(n)\end{array}$ & $\begin{array}{l}\text { Gout concern } \\
\text { overall }\end{array}$ & $\begin{array}{l}\text { Gout medication } \\
\text { side effects }\end{array}$ & $\begin{array}{l}\text { Unmet gout } \\
\text { treatment need }\end{array}$ & $\begin{array}{l}\text { Well being } \\
\text { during attack }\end{array}$ & $\begin{array}{l}\text { Gout concern } \\
\text { during attack }\end{array}$ \\
\hline \multicolumn{6}{|c|}{ Attack-related characteristics } \\
\hline \multicolumn{6}{|l|}{$\begin{array}{l}\text { Number of attacks } \\
\text { in the past year }\end{array}$} \\
\hline Zero $(n=56)$ & $36.7(24.7)$ & $37.7(26.4)$ & $26.3(19.2)$ & $55.3(29.0)$ & $42.7(21.7)$ \\
\hline $\mathrm{I}-2(\mathrm{n}=87)$ & $56.6(26.4)$ & $43.8(22.3)$ & $31.5(19.4)$ & $54.3(25.5)$ & $43.9(23.5)$ \\
\hline $3-5(n=83)$ & $73.9(2 \mid .2)$ & $52.0(24.0)$ & $45.0(18.1)$ & $56.6(23.7)$ & $58.4(21.6)$ \\
\hline $6-10(n=24)$ & $77.6(21.0)$ & $62.5(29.0)$ & $39.2(13.8)$ & $56.1(26.0)$ & $56.5(21.6)$ \\
\hline$>10(n=35)$ & $82.3(20.8)$ & $56.4(26.7)$ & $56.4(23.8)$ & $60.2(29.4)$ & $52.9(29.2)$ \\
\hline$P$ value & $P<0.001$ & $P<0.001$ & $P<0.001$ & $P=0.858$ & $P<0.001$ \\
\hline $\begin{array}{l}\text { Gout pain Typical } \\
\text { attack (VAS 0-10) }\end{array}$ & $\begin{array}{l}0.30 I \\
(n=16 I)\end{array}$ & $\begin{array}{l}-0.066 \\
(n=160)\end{array}$ & $\begin{array}{l}0.066 \\
(n=157)\end{array}$ & $\begin{array}{l}0.494 \\
(n=158)\end{array}$ & $\begin{array}{l}0.308 \\
(n=|6|)\end{array}$ \\
\hline $\begin{array}{l}\text { Number joints involved } \\
\text { in Typical attack }\end{array}$ & $\begin{array}{l}0.280 \\
(n=167)\end{array}$ & $\begin{array}{l}0.182 \\
(n=167)\end{array}$ & $\begin{array}{l}0.217 \\
(n=164)\end{array}$ & $\begin{array}{l}0.245 \\
(n=165)\end{array}$ & $\begin{array}{l}0.184 \\
(n=167)\end{array}$ \\
\hline \multicolumn{6}{|l|}{ Overall gout characteristics } \\
\hline \multicolumn{6}{|l|}{$\begin{array}{l}\text { Time with gout pain } \\
\text { between attacks }\end{array}$} \\
\hline All of the time $(n=20)$ & $88.4(18.8)$ & $53.1(25.6)$ & $51.7(20.7)$ & $76.3(20.0)$ & $68.4(23.6)$ \\
\hline Most of the time $(n=27)$ & $79.2(20.7)$ & 6I.I (25.8) & $50.6(20.1)$ & $67.3(19.0)$ & $63.4(21.1)$ \\
\hline Some of the time $(n=57)$ & $73.8(2 \mid .7)$ & $56.6(25.5)$ & $43.3(19.9)$ & $58.7(26.5)$ & $52.2(23.2)$ \\
\hline A little of the time $(n=58)$ & $65.4(23.9)$ & $46.3(23.4)$ & $36.0(17.3)$ & $49.4(24.4)$ & $49.1(24.5)$ \\
\hline None of the time $(n=1 \mid 8)$ & $47.7(28.1)$ & $40.6(24.6)$ & $31.3(21.1)$ & $51.5(26.8)$ & $42.7(22.0)$ \\
\hline$P$ value & $P<0.001$ & $P<0.001$ & $P<0.001$ & $P<0.00 I$ & $P<0.001$ \\
\hline \multicolumn{6}{|l|}{ Presence of tophi } \\
\hline Yes $(n=4 I)$ & $68.4(30.6)$ & $54.3(29.0)$ & $45.4(21.7)$ & $58.6(29.2)$ & $56.7(26.7)$ \\
\hline No $(n=122)$ & $60.0(28.5)$ & $46.3(25.4)$ & $37.1(20.6)$ & $58.8(25.2)$ & $50.0(22.9)$ \\
\hline$P$ value & $P=0.111$ & $P=0.096$ & $P=0.029$ & $P=0.960$ & $P=0.124$ \\
\hline Latest serum uric acid & $0.240(n=166)$ & $0.028(n=166)$ & $0.153(n=165)$ & $0.014(n=162)$ & $0.062(n=166)$ \\
\hline
\end{tabular}

Notes: Correlations are for descriptive purposes and were not therefore analyzed for significance; ANOVAS were performed for categorical variables. Pearson correlations performed for continuous variables.

Abbreviations: ANOVA, analysis of variance; SD, standard deviation; VAS, Visual Analogue Scale. 
Table 6 Factors associated with gout impact (adjusted for age, gender, number of comorbidities)

\begin{tabular}{|c|c|c|}
\hline Dependent variable & Covariates & $\begin{array}{l}\text { Partial corr. } \\
\text { coefficient }\end{array}$ \\
\hline \multirow[t]{6}{*}{ Gout concern overall } & Number of attacks past year & $0.483 * *$ \\
\hline & Gout pain typical attack & $0.318 * *$ \\
\hline & Number of joints involved typical attack & $0.253^{* *}$ \\
\hline & Time with gout pain between attacks & $0.467 * *$ \\
\hline & Presence of tophi & $-0.175^{*}$ \\
\hline & Latest serum uric acid level & $0.188 *$ \\
\hline \multirow[t]{6}{*}{ Gout medication side effects } & Number of attacks past year & $0.253 * *$ \\
\hline & Gout pain typical attack & -0.042 \\
\hline & Number of joints involved typical attack & $0.202 *$ \\
\hline & Time with gout pain between attacks & $0.246 * *$ \\
\hline & Presence of tophi & -0.145 \\
\hline & Latest serum uric acid level & -0.011 \\
\hline \multirow[t]{6}{*}{ Unmet gout treatment need } & Number of attacks past year & $0.4 \mathrm{I} \mathrm{I}^{* *}$ \\
\hline & Gout pain typical attack & 0.035 \\
\hline & Number of joints involved typical attack & $0.169 *$ \\
\hline & Time with gout pain between attacks & $0.346 * *$ \\
\hline & Presence of tophi & $-0.197^{*}$ \\
\hline & Latest serum uric acid level & 0.092 \\
\hline \multirow[t]{6}{*}{ Well being during attack } & Number of attacks past year & -0.013 \\
\hline & Gout pain typical attack & $0.517 * *$ \\
\hline & Number of joints involved typical attack & $0.223 * *$ \\
\hline & Time with gout pain between attacks & $0.247 * *$ \\
\hline & Presence of tophi & -0.042 \\
\hline & Latest serum uric acid level & -0.004 \\
\hline \multirow[t]{6}{*}{ Gout concern during attack } & Number of attacks past year & $0.154^{*}$ \\
\hline & Gout pain typical attack & $0.308 * *$ \\
\hline & Number of joints involved typical attack & 0.129 \\
\hline & Time with gout pain between attacks & $0.310 * *$ \\
\hline & Presence of tophi & -0.169 \\
\hline & Latest serum uric acid level & 0.056 \\
\hline
\end{tabular}

Notes: *Significant at the 0.01 level (2-tailed); **Significant at the 0.05 level (2-tailed). Partial correlations were performed between each GIS scale and I) Number of attacks in past year and time with gout pain between attacks $(n=271), 2)$ Pain severity of typical gout attack and Number of joints involved in typical attack $(n=149)$, and 3) Latest sUA level and presence of tophi $(n=129)$. Differences in number of patients for each correlation due to missing item responses.

correlation was observed with "well being during an attack" $(r=0.517)$. Notably, there was only minimal correlation between the presence of tophi and GIS scales, although a significant difference in the "unmet gout treatment need" scale had been detected using the unadjusted ANOVA (Table 5).

\section{Discussion}

This is the first study to use a gout-specific HRQoL instrument, the GIS, to measure the association of gout characteristics with HRQoL. A large degree of variation in GIS scale scores was observed in this cross-sectional community patient sample. This would be expected given the diversity in gout characteristics (attack and overall) in the study sample. On average, patients indicated they were concerned about the impact of gout on their HRQoL despite the fact that virtually all had prescribed medications for their gout and most believed their treatment was effective (low "unmet gout treatment need" scores). Whether this reflects inadequate treatments or low expectations for treatment effect or some other explanation cannot be determined from our study but warrants further investigation. The three remaining GIS scores had values near the scale midpoint indicating a level of gout interference with activities that was moderate, some of the time.

Frequency of attacks showed a consistent and linear relationship with patients' assessment of their HRQoL using 
the gout-specific GIS scales. This was a similar finding to the only other studies examining the effect of gout characteristics on HRQoL which also reported frequency of attacks to be significantly related to reduced HRQoL using general QOL scales (SF-36). ${ }^{12,14}$ In addition to attack frequency, the amount of time with gout pain between attacks was also consistently associated with lower HRQol measured by GIS scales. As may be expected, the amount of pain during an attack was associated most strongly with the two during attack GIS scales ("well-being during attack" and "gout concern during attack") and the "gout concern overall" scale. Differences in GIS scale scores among patients with varying degrees of gout activity were pronounced. For example, patients with three to five attacks in the past year reported GIS scale values from 14 to 36 points higher (greater gout impact) than patients with no attacks in the past year for four of the five GIS scales. Similarly, patients indicating they experienced gout pain between attacks "some of the time" reported GIS scale values from 7 to 25 points higher than patients with no gout pain between attacks. Attack frequency, pain during an attack and gout pain between attacks remained important correlates of gout-specific HRQoL even after controlling for age, gender, and comorbidities. Unlike previous studies, we found a low correlation of the number of joints involved in a typical attack, sUA level and presence of tophi with all GIS scales suggesting these common, objective measures of gout may not be good indicators of the impact of gout on the patients' HRQoL.

There are several limitations of this study that should be considered when interpreting results. Patients were primarily Caucasian and recruited from arthritis and primary care clinics in three US cities, thus our results may not be generalizable to other races, geographic areas, or to patients not seeking health care for their gout. This was a cross-sectional, questionnaire-based study which relied on patient report which may be subject to recall bias. Physicians were contacted after patients completed their questionnaire therefore the report of presence or absence of tophi and most recent sUA level, although aided by the patient's chart to lessen recall bias, was not representative of the same point in time as patient questionnaire data. This may be a particularly important limitation for sUA level which varies over time and with the patient's gout condition; thus the association between GIS scores and sUA observed in this study may be different in a patient group completing the GIS at the same time as sUA testing. Interpretation of the magnitude of GIS scores was necessarily limited and based on clinical judgment of the authors since this was the first sample of patients to complete the GIS. A prospective study, in which clinical and patient response data are collected concurrently, is currently ongoing to test the responsiveness and determine the minimal important difference for each GIS scale. Also since this is the first sample of patients to complete the GIS, comparison of scale scores to other gout patient samples is not possible at this time. The GIS scores reported in this study may be considered as providing benchmarks for future studies.

\section{Conclusion}

This is the first study to use a gout-specific instrument, the Gout Impact Scale (GIS), to assess the association of gout characteristics with patients' HRQoL. Attack frequency, pain during an attack and gout pain between attacks were important correlates of patients' ratings of their gout-specific HRQoL. Common objective measures such as sUA level, presence of tophi and the number of joints involved in a typical attack did not appear to be good indicators of the impact of gout on the patients' HRQoL. Further studies are needed to determine the minimal important difference for each GIS scale and interpret our results relative to other patient populations with gout.

\section{Acknowledgments/disclosures}

This study was sponsored in part by Takeda (formerly TAP) Pharmaceutical, Inc. and the University of California, San Diego General Clinical Research Center Program, M01 RR00827, National Center for Research Resources, National Institutes of Health and the VA Research Service. DK was also supported by a National Institutes of Health Award (NIAMS K23 AR053858-01A1) during this period. JDH has received a research grant from TAP and Takeda pharmaceuticals and consultant fees from Regeneron. RT is a consultant for Takeda, Savient, EnzymeRx, Altus, BioCryst, Regeneron, Novartis, Pfizer, URL Pharma, and Proctor and Gamble and has received grants from the Research Service of the Department of Veterans Affairs and Takeda Pharmaceuticals. DK has received consultant fees and research grants from Takeda Pharmaceuticals. JAS has received research grants from TAP pharmaceuticals for this and other research projects. All other authors have declared no conflicts of interest.

\section{References}

1. Kim KY, Schumacher HR, Hunsche E, et al. A literature review of the epidemiology and treatment of acute gout. Clin Ther. 2003;25: 1593-1617.

2. Adams PF, Hendershot GE, Marano MA. Centers for Disease Control and Prevention/National Center for Health Statistics. Current estimates from the National Health Interview Survey, 1996.Vital Health Stat. 1999;10(200):1-203. 
3. Osterhaus JT, Patel P, Palo W, et al. Patient reported outcomes associated with gout: Baseline results from two clinical trials. San Diego, CA: Presented at the Annual Meeting of the American College of Rheumatology; 2005.

4. Alvarez-Nemegyei J, Cen-Pisté JC, Medina-Escobedo M, et al. Factors associated with musculoskeletal disability and chronic renal failure in clinically diagnosed primary gout. J Rheumatol. 2005;32:1923-1927.

5. Vázquez-Mellado J, Cruz J, Guzman S, et al. Severe tophaceous gout. Characterization of low socioeconomic level patients from Mexico. Clinl Exp Rheumatol. 2006;24(3):233-238.

6. Geletka RC, Hershfield MS, Scarlett THE, et al. Severe gout is associated with impaired quality of life and functional status [abstract]. Arthritis Rheum. 2004;50(9)(suppl):S340-S341.

7. Khanna D, Mansoor A, Yontz D, et al. The disutility of chronic gout. Qual Life Res. 2008;17(5):815-822.

8. Khanna D, Mansoor A, Yontz D. et al. Willingness to pay for a cure in patients with chronic gout. Med Decis Making. 2008;28(4):606-613.

9. National Institute for Health and Clinical Excellence. Febuxostat for the management of hyperuricaemia in people with gout. Available from: http://www.nice.org.uk/nicemedia/pdf/STAFebuxostatGoutFAD.pdf. Accessed January 12, 2009.

10. Taylor WJ, Schumacher HR, Singh JA. et al. Assessment of outcome in clinical trials of gout - a review of current measures. Rheumatology. 2007;46(12):1751-1756.

11. Taylor WJ, Schumacher HR Jr, Baraf HS, et al. A modified Delphi exercise to determine the extent of consensus with OMERACT outcome domains for studies of acute and chronic Gout. Ann Rheum Dis. 2008;67:888-891.
12. Sundy JD, Schumacher HR, Becker MA, et al. Quality of life in patients with treatment failure gout. Ann Rheum Dis. 2006;65(Suppl II):271.

13. Geletka RC, Hershfield MS, Scarlett EL, et al. Severe gout is associated with impaired quality of life and functional status. Arthritis Rheum. 2004;50:S340-S341.

14. Lee SJ, Hirsch JD, Terkeltaub R, et al. Perceptions of disease and health-related quality of life among patients with gout. Rheumatology. 2009;48(5):582-586.

15. Picavet HJ, Hoetmans N. Health related quality of life in multiple muscoloskelatal diseases: Sf-36 and EQ-5D in the DMC3 study. Ann Rheum Dis. 2004;63:723-729.

16. Roddy E, Zhang W, Doherty M. Is gout associated with reduced quality of life? A case-control study. Rheumatology. 2007;46:1441-1444.

17. Singh J, Strand V. Gout is associated with more comorbidities, poorer health related quality of life and higher health care utilization in US veterans. Ann Rheum Dis. 2008;67(9):1310-1316.

18. Bootman JL, Townsend RJ, McGhan WF, editors. Principles of pharmacoeconomics, 3rd edition. Cincinnati, OH: Harvey Whitney Books Company; 2005:157.

19. Hirsch JD, Lee SJ, Terkeltaub R, et al. Evaluation of an instrument assessing gout association on health-related quality of life. J Rheumatol. 2008;35(12):2406-2414.

20. Cohen J, Cohen P, West SG, et al. Applied Multiple Regression/ Correlation Analysis for the Behavioral Sciences, 2nd edition. Hillsdale, NJ: Lawrence Erlbaum Associates; 2003.
Patient Related Outcome Measures

\section{Publish your work in this journal}

Patient Related Outcome Measures is an international, peer-reviewed, open access journal focusing on treatment outcomes specifically relevant to patients. All aspects of patient care are addressed within the journal and practitioners from all disciplines are invited to submit their work as well as healthcare researchers and patient support groups. Areas covered will

\section{Dovepress}

include: Quality of life scores; Patient satisfaction audits; Treatment outcomes that focus on the patient; Research into improving patient outcomes; Hypotheses of interventions to improve outcomes; Short communications that illustrate improved outcomes; Case reports or series that show an improved patient experience; Patient journey descriptions or research. 